\title{
FPGA Implementation of Single Bit Error Correction using CRC
}

\author{
Pramod S P \\ Department of ECE, \\ DSCE,VTU,Bangalore,India
}

\author{
Rajagopal A \\ Department of E\&C, DSCE, \\ VTU,Bangalore, INDIA
}

\author{
Akshay S Kotain \\ Department of E\&C, DSCE, \\ VTU,Bangalore, INDIA
}

\begin{abstract}
Transferring data between two points is very essential, also the accuracy of the transferred data is vital for some critical applications, but an error during the transmission of data is very common. The Cyclic Redundancy Check (CRC) method is generally used for error detection and correction. In this paper, we have proposed a new technique for error detection and correction in case of CRC-16, which is hardware optimized and works at relatively higher frequency and speed. In the proposed method, it is possible to detect the exact place of single bit error and correct them using minimum hardware. This method involves no look tables and hence is memory efficient. This paper focuses on effective implementation of this method on FPGA.
\end{abstract}

\section{Keywords}

CRC, Field Programmable Gate Array, Single bit error correction, Parallelism, non-lookup technique.

\section{INTRODUCTION}

The Internet is growing rapidly in terms of number of users and amount of bandwidth used. Besides the transmission and switching speeds, the per-packet operations necessary for internet Protocol (IP) packet forwarding are the current limiting factors. As transmission speeds are continually increasing, IP packet processing overheads have become the main bottleneck [5]. The received data may not be same as the transmitted data because of noise and interference, which leads to errors during the process of data transmission and storage. Hence, to achieve better quality data transmission, coding and detection methods are employed. CRC is very simple and efficient method to detect and correct the errors. A Cyclic Redundancy Check (CRC) is a type of function that takes an input data stream of any length, and produces an output value of 32-bit or 24 bit integer. Single bit error correction using look up table technique was presented in [3] and [4]. A CRC can be used as a checksum to detect accidental alteration of data during transmission or storage. A CRC theoretically can correct all single, double and odd bits in error [6]. The process of encoding and decoding are discussed in the following sections.

In this paper, we have introduced a new approach for the error detection and correction using non-look up table technique based on FPGA, in case of CRC. This approach is memory efficient and operates at high frequency. Since most of the methods currently employed are based on look up table technique, they consume more space and also there will be a reduction in speed because of the overhead required in accessing the ROM which stores the look up table. We will then review the Sunil Shukla [1] method that corrects single bit error and also other look up table optimization techniques presented in [2]. We have also discussed a method which makes the algorithm hardware implementable and the results obtained are tabulated.

\section{PRINCIPLE}

$\mathrm{CRC}$ is one of the most famous and robust error control methods. Its computation resembles a long division operation in which the quotient is discarded and the remainder becomes the result, with the important distinction that the arithmetic used is the carry-less arithmetic of a finite field or Modulo-2 addition. The length of the remainder is always less than or equal to the length of the divisor, which therefore determines how long the result can be.

In this method, the receiver divides the data by generator polynomial and then gets the remainder. The receiver then uses parallelism technique to correct single bit error by a shifter circuit which will be explained in the following sections. This improvised proposed method requires zero memory and minimum hardware.

Before transmission, the frame produced by CRC encoder consists of check bits cascaded with data bits. These check bits which are redundant are used for error detection and correction at the receiver. For the calculation of checksum bits, a fixed generator polynomial is chosen. The data bits are then divided with the generator polynomial and the resultant remainder is the checksum bits. The division of the data by the generator polynomial is performed using the circuit which is shown in Figure 1.

\section{CRC-16 Generator Polynomial $G(x)=x^{16}+x^{12}+x^{5}+1$}

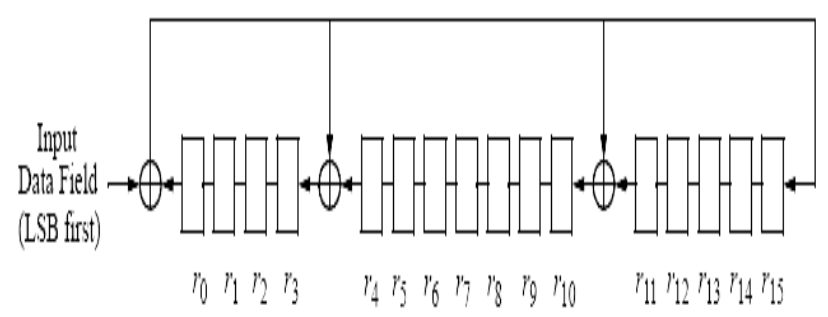

Fig 1: Polynomial divider.

Initially, all the remainder registers are initialized to zero. Then the data bits are shifted into the circuit with the order such that the LSB of data is entered into the circuit first. Now depending upon the generator polynomial, the data is just shifted right or it is added with the feed- back value. Modulo2 addition is performed. Once all the data bits are shifted into the registers, the remainder registers indicates the checksum bits. These checksum bits are cascaded with the data bits before the data is transmitted.

The codeword $\mathrm{C}(\mathrm{x})$ can be calculated using Equation 1.

$$
C(x)=\frac{x^{n-k_{*}}(x)}{G(x)}+D(x)
$$


The first part of right hand side of Equation 1 represents the checksum bits. Here ' $n$ ' represents the frame width and ' $k$ ' represents the data width. As an example taking $n=24$ and $\mathrm{k}=8$ the calculation of checksum bits is shown below.

$$
\begin{gathered}
x^{16}+x^{12}+x^{5}+1 \frac{x^{5}+x^{4}+x^{2}+x}{x^{21}+x^{20}+x^{18}+x^{16}} \\
\frac{x^{21}+x^{17}+x^{10}+x^{5}}{x^{20}+x^{18}+x^{17}+x^{16}+x^{10}+x^{5}} \\
\frac{x^{20}+x^{16}+x^{9}+x^{4}}{x^{18}+x^{17}+x^{10}+x^{9}+x^{5}+x^{4}} \\
x^{18}+x^{14}+x^{7}+x^{2} \\
x^{17}+x^{14}+x^{10}+x^{9}+x^{7}+x^{5}+x^{4}+x^{2} \\
x^{17}+x^{13}+x^{6}+x^{2} \\
x^{14}+x^{13}+x^{10}+x^{9}+x^{7}+x^{6}+x^{5}+x^{4}+x^{2}+x
\end{gathered}
$$

Here:

Data, D(x):00010011

Check bits:00110011011110110

Codeword, C(x):00110011011110110_00010011

Similarly the codeword for CRC-16 can be calculated. The CRC Encoder is designed to generate the codeword in a single clock cycle with high speed and efficiency.

\section{Frame Structure of CRC-16:}

\begin{tabular}{l|l} 
Check bits (32 down to 17$)$ & Data bits(16 down to 1$)$
\end{tabular}

\section{Frame Structure of CRC-8:}

\begin{tabular}{|l|l} 
Check bits (24 down to 9) & Data bits(8 down to 1$)$
\end{tabular}

\section{PROPOSED METHOD FOR ERROR DETECTION AND CORRECTION}

In this paper, we will be presenting a unique way of implementing multiple bit error detection and single bit error correction using CRC for a frame width of 24 bits and 32 bits. Let Ftr be the frame transmitted in which the checksum is appended after 16 or 8 bits of data. We can express Ftr as shown in Equation 2.

$$
\text { Ftr }=\text { Dtr \& Ctr }
$$

Where,

\& - concatenation operator.

Dtr- transmitted 16 or 8 bit data.

$\mathrm{Ctr}$ - transmitted 16 bit checksum.

At the receiver side, let Fre be the received frame as shown in Equation 3.

$$
\text { Fre }=\text { Dre } \& \text { Cre }
$$

Where,

Cre indicates received checksum.
Dre represents received data.

Receiver again calculates CRC on the received data. Let Ccal indicates the CRC calculated over Dre at the receiver side. If no error has occurred during transmission then Cre and Ccal are equal. But if some bit(s) are in error, then Cre and Ccal will be in mismatch. In such cases the error needs to be detected and corrected .Hence we need to calculate the syndrome which is given by:

$$
\text { Syn=Cre XOR Ccal }
$$

If the syndrome is calculated as proposed in [9] which uses a circuit similar to the circuit as shown in Figure 1, only with more number of registers, then it requires as many clock cycles as the number of bits in the codeword. But by using equation 4 , the syndrome can be calculated in minimum number of clock cycles. This method of syndrome calculation was proposed in [1] and is an efficient one, hence we have adopted it.

Table 1. Possible number of error patterns

\begin{tabular}{|c|c|}
\hline Standard & Single Bit Error \\
\hline CRC- 8 & 24 \\
\hline CRC-16 & 32 \\
\hline
\end{tabular}

This paper uses two standards CRC-16 and CRC-8 for correcting single bit errors. Here we have designed the algorithm to detect more than one error and we will raise a flag indicating retransmission of the frame if there were more than one error.

There are 2 cases in which the single bit error can occur. In the first case, one bit error can occur in the data bits. In the second case, one bit error can be in checksum bits. Hence the total number of possible single bit errors in data for both the standards is shown in Table 1.

For CRC-16 if ' $\mathrm{i}$ ' is the position of the error then for case 1) $1 \leq \mathrm{i} \leq 16$ and for case 2$) \quad 17 \leq \mathrm{i} \leq 32$. Similarly for CRC- 8 only the limits will change.

In our proposed method we have used a new technique for detecting errors in both the cases (described in previous paragraph). This method is an outcome of the fact that if an error occurs as in case 2 then the syndrome pattern will have 1 's equal to no. of bits in error. As we know, we only have to correct the error in the data bits, there is no need to correct the error which has occurred in the checksum bits. But we need to detect the errors in the checksum bits. This method of analysis is advantages since it reduces memory requirements by $76 \%$ and also we need to worry only about the errors in data, this reduces the load on the computational block of the receiver.

The analysis of the two cases mentioned above is explained in detail by using tables. The syndrome pattern for Case 2 for CRC-16 is shown in Table 2. This pattern is similar even for CRC-8. As we can see the pattern consists of only bit with logical level 1 . Similarly Table 3 represents the syndrome patterns of the Case 1 for CRC-16 and CRC- 8 standards.

In [1] only lower 8 bits are used for addressing leading to 256 memory locations. Also, the no. of patterns to be checked in [1] is only 32 . Hence the above type of addressing is not applicable. Here we require the entire 16 bits of the syndrome for locating and correcting the data. This results in 65536 memory locations. Considering a ROM with 65536 or 256 memory locations is not so efficient when compared to our 
method of not using any memory block at all. We can analyze the syndrome more effectively without using any memory. The use of ROM [8] also introduces complexity into the design, since we need to constantly read error patterns from it and to take care of correct timing.

Table 2.

Syndrome patterns of single bit error in check bits

\begin{tabular}{|c|c|}
\hline Syndrome pattern & $\begin{array}{c}\text { Location of the bit in } \\
\text { error (i) }\end{array}$ \\
\hline 0000000000000001 & 17 \\
\hline 0000000000000010 & 18 \\
\hline 0000000000000100 & 19 \\
\hline 0000000000001000 & 20 \\
\hline 0000000000010000 & 21 \\
\hline 0000000000100000 & 22 \\
\hline 0000000001000000 & 23 \\
\hline 0000000010000000 & 24 \\
\hline 0000000100000000 & 25 \\
\hline 0000001000000000 & 26 \\
\hline 0000010000000000 & 27 \\
\hline 0000100000000000 & 28 \\
\hline 0001000000000000 & 29 \\
\hline 0010000000000000 & 30 \\
\hline 0100000000000000 & 31 \\
\hline 1000000000000000 & 32 \\
\hline
\end{tabular}

To explain the method of correcting single bit error that we have proposed let us consider an example of $(7,4)$ CRC code as in [9]. The syndrome generator circuit for this codeword is shown Figure. 2. This circuit of shift registers is similar to the circuit shown in Figure 1 except that it has only three shift registers corresponding to three check bits in the codeword.

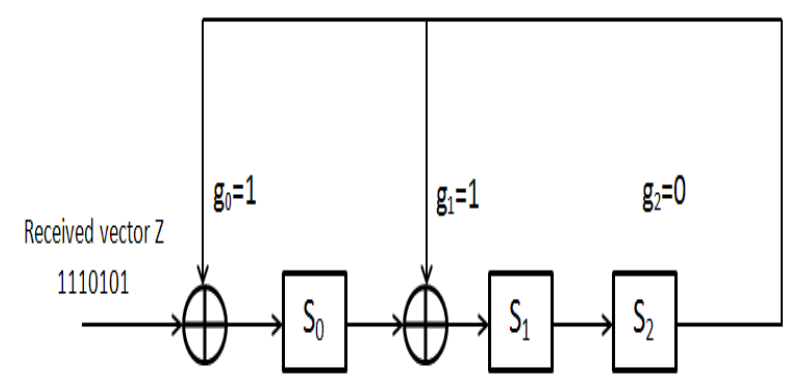

Fig 2: syndrome circuit for $(7,4)$

Here the generator polynomial used is $g(x)=1+x+x^{3}$. The received vector $Z=1110101$ with three check bits and a nibble of data. In this codeword first three MSB bits are check bits which is concatenated with a nibble of data. Here the 3rd MSB or 3rd Check bit is in error. So we need to detect it and correct it. In conventional method the output of the circuit in Figure 2 which is syndrome is used to address a look up table which actually stores the error pattern. This error pattern is then XORed with the CRC frame to get the correct data.
Table 3.

Syndrome patterns of single bit error in data bits

\begin{tabular}{|c|c|c|}
\hline Syndrome pattern & $\begin{array}{c}\text { The bit in } \\
\text { error for } \\
\text { CRC-8 }\end{array}$ & $\begin{array}{c}\text { The bit in } \\
\text { error for } \\
\text { CRC-16 }\end{array}$ \\
\hline 1000010000001000 & 8 & 16 \\
\hline 0100001000000100 & 7 & 15 \\
\hline 0010000100000010 & 6 & 14 \\
\hline 0001000010000001 & 5 & 13 \\
\hline 1000110001001000 & 4 & 12 \\
\hline 0100011000100100 & 3 & 11 \\
\hline 0010001100010010 & 2 & 10 \\
\hline 0001000110001001 & 1 & 9 \\
\hline 1000110011001100 & - & 8 \\
\hline 0100011001100110 & - & 7 \\
\hline 0010001100110011 & - & 6 \\
\hline 1001010110010001 & - & 5 \\
\hline 1100111011000000 & - & 4 \\
\hline 0110011101100000 & - & 3 \\
\hline 0011001110110000 & - & 2 \\
\hline 0001100111011000 & - & 1 \\
\hline & & \\
\hline
\end{tabular}

But, in the proposed method the same circuit in Figure 2 is used but more number of clock cycles are required. That is when all the 7 received bits are entered into the syndrome calculator, ' 0 's are now fed into it, from 8th shift onwards as shown Table 4. Each time a ' 0 ' is fed into the circuit, the shift register contents are tabulated. This process of feeding ' 0 's continues till the shift register contents read S0 S1 S2 $=100$. In general for $(n-k)$ shift register, the contents should read S0, $\mathrm{S} 1 \ldots \ldots . \mathrm{Sn}-\mathrm{k}-1=100 \ldots \ldots \ldots . . .0$. i.e., 1 followed by $(\mathrm{n}-\mathrm{k}-1)$ number of 0 s. In Table (4), we find that at the 12th shift we get shift register contents as 100 . The error is then located and corrected as given below.

Table 4.

Contents of shift register in the syndrome calculator

\begin{tabular}{|l|c|c|c|c|}
\hline \multirow{2}{*}{$\begin{array}{l}\text { No. of } \\
\text { shifts }\end{array}$} & \multirow{2}{*}{$\begin{array}{c}\text { Input } \\
\mathbf{Z}(\mathbf{x})\end{array}$} & \multicolumn{3}{|c|}{ Shift Register Contents } \\
\cline { 3 - 5 } & $\mathbf{S}_{\mathbf{0}}$ & $\mathbf{S}_{\mathbf{1}}$ & $\mathbf{S}_{\mathbf{2}}$ \\
\hline \multirow{2}{*}{$\begin{array}{c}\text { Initialization and } \\
\text { LSB of } \mathbf{Z}(\mathbf{x}) \text { enter } \\
\text { first }\end{array}$} & \multicolumn{3}{|c|}{$\begin{array}{c}\text { Shift register contents are } \\
\text { cleared }\end{array}$} \\
\cline { 3 - 5 } & 0 & 0 & 0 \\
\hline 1 & 1 & 1 & 0 & 0 \\
\hline 2 & 0 & 0 & 1 & 0 \\
\hline 3 & 1 & 1 & 0 & 1 \\
\hline 4 & 0 & 1 & 0 & 0 \\
\hline 5 & 1 & 1 & 1 & 0 \\
\hline 6 & 1 & 1 & 1 & 1 \\
\hline 7 & 1 & 0 & 0 & 1 \\
\hline 8 & 0 & 1 & 1 & 0 \\
\hline 9 & 0 & 0 & 1 & 1 \\
\hline 10 & 0 & 1 & 1 & 1 \\
\hline 11 & 0 & 1 & 0 & 1 \\
\hline 12 & 0 & 1 & 0 & 0 \\
\hline
\end{tabular}


As we can see from Table (4) after 7th shift we get the syndrome from the circuit in "fig 2 ". Since this syndrome is not equal to " 000 " it indicates an error. Then the procedure is continued as explained earlier until 12th shift when shift register content is "100". This shift number indicates the position of the error as shown below.

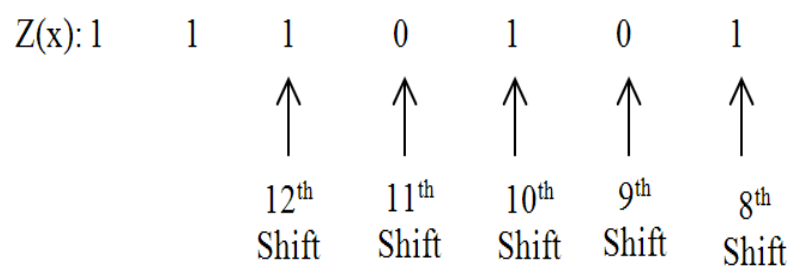

The 5th bit counting from right is in error.

Therefore Error pattern is $\mathrm{E}=0010000$

$$
\text { Corrected vector } \begin{aligned}
\mathrm{V} & =\mathrm{Z} \text { XOR E } \\
& =1110101 \text { XOR } 0010000 \\
\mathrm{~V} & =1100101
\end{aligned}
$$

This is the same method employed in correcting single bit error in CRC-8 and CRC-16.

\section{IMPLEMENTATION ON FPGA}

A parallel VLSI architecture of the decoder circuitry is as shown in "Fig.3". The syndrome calculator generates the vector syndrome from the received frame based on the circuit similar to "fig 1". This operation requires 32 clock cycles and hence this timing is kept track by a Timing and Control Unit with the help of a 5 bit counter. Then if the syndrome is all zero, it means that the corrected data is same as received data or else if the number of 1's in the syndrome pattern is equal to 1 , it indicates an error in the check bits. Finally, if single bit error is present in data bits which is indicated by the above equations not being satisfied then 0 's are input into the syndrome calculator circuit and after each 0 input the vector syndrome is XORed with the pattern " 0000000000000001 " and checked whether the result is zero or not. If it is zero then the corrected data is obtained by XORing the received data with the error pattern register content. If not then the procedure is continued and error pattern register content is shifted towards left by 1 bit. The initial content of error pattern register is " 0000000000000001 ".

The Timing and Control Unit is designed using a state diagram as shown in "fig 4". It consists of four states, initially in S0 we need to compute the syndrome. Hence we need a delay of 32 clock cycles and received bits must be routed to syndrome calculator circuit. This is achieved by setting the mode pin to high and Sel1 pin to low which in-turn connects clk input to the 5 bit counter and the CRC frame to the syndrome circuit respectively. So when the Top signal of the counter becomes 1 , it indicates the completion of the delay required. Then the state transition to $\mathrm{S} 1$ occurs where mode pin turned low so that the counter can count the number of 1 's in the syndrome vector. The counter content $\mathrm{C}$ is sent back to the control unit. Concurrently the vector syndrome is checked whether or not it is equal to 0 using generic NOR gate. If true $\mathrm{NbE}$ signal goes high. State jumps to $\mathrm{S} 2$ if $\mathrm{C}=1$ or $\mathrm{NbE}=1$ and the select pin Sel2 is made low which makes the mux connect received data to corrected data. If $\mathrm{C} \neq 1$ and $\mathrm{NbE}=0$ then the control jumps from $\mathrm{S} 1$ to $\mathrm{S} 3$ which indicates that a single bit error is present in data bits. In S3 the control unit sets the Sell to high which inputs 0's into the syndrome calculator. After each clock cycle syndrome is XORed with " $10000 \ldots . . .0$ " and checked if zero using the same Generic NOR gate. If true $\mathrm{SbE}$ goes high indicating the completion of the process, if false then error pattern register is shifted towards left as explained in previous section. The control unit stays in $\mathrm{S} 3$ until $\mathrm{SbE}$ is 0 . Once $\mathrm{SbE}$ is 1 then $\mathrm{Sel} 2$ is set as 0 to connect the output corrected data to a pattern obtained by XORing received data with content of error pattern register.

The architecture for CRC-8 is similar to Figure 3" but it requires a extra comparator circuit after 5 bit counter since initially we need a delay of 24 clock cycles and hence Top signal cannot be used. So we use a comparator to check if counter has reached 24 or not.

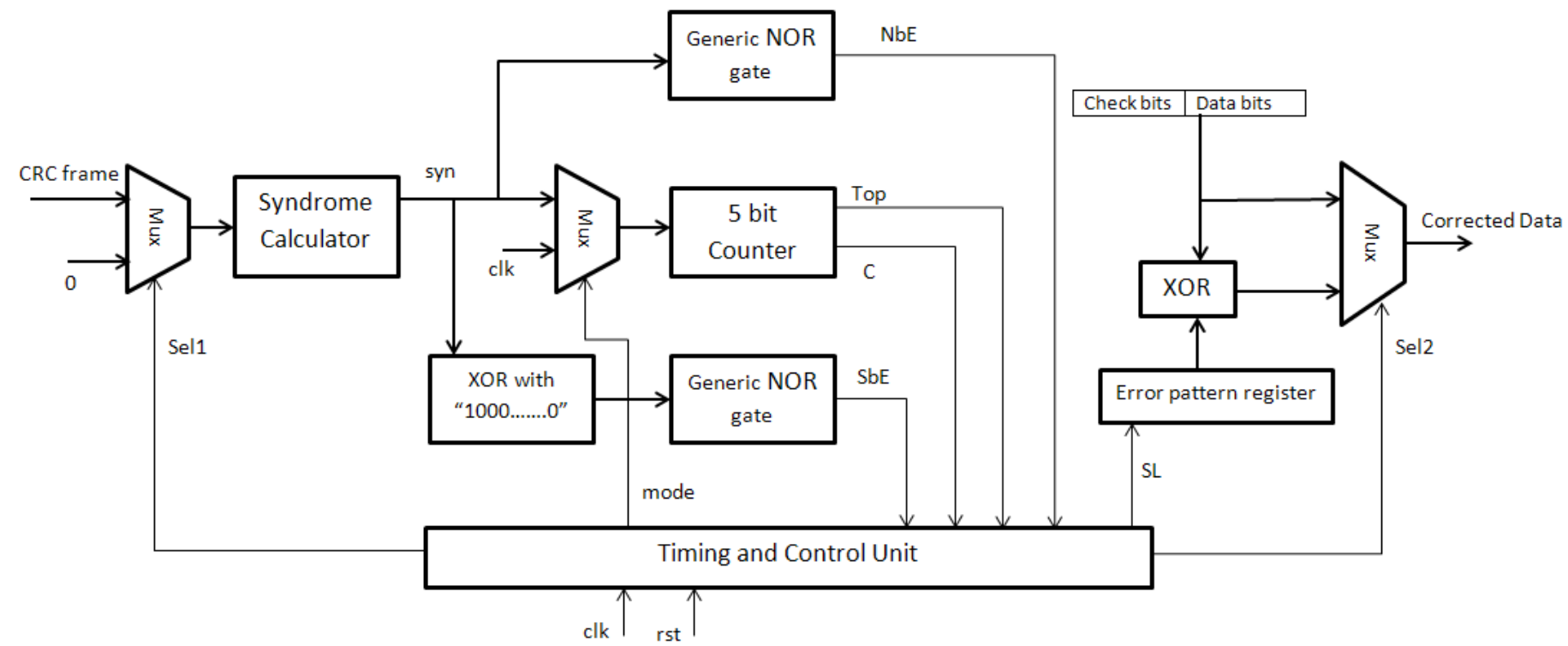

Fig 3: VLSI Architecture 


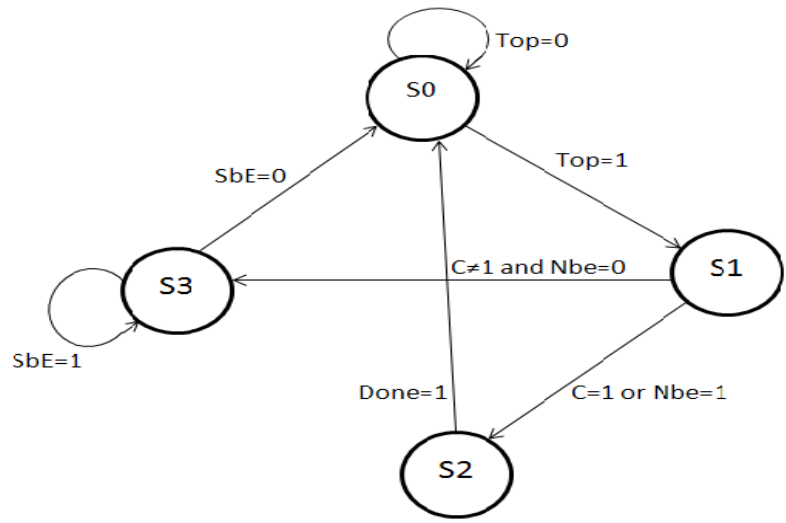

Fig 4: state diagram of the control unit

The algorithm has been implemented and verified on Xilinx Virtex-5 FPGA device. The code was written in VHDL and synthesized using XST (Xilinx synthesis tool). The device used for implementation is $x c 5 v l x 30$ with speed grade 3 . The results obtained are summarized in Table 5. Simulation is carried out using MODELSIM SE 6.5 and the simulation results are shown in Figure 5.

Table 5. Device utilization of FPGA

\begin{tabular}{|c|c|c|c|c|}
\hline \multirow{2}{*}{ Features } & \multicolumn{2}{|c|}{ CRC-8 } & \multicolumn{2}{c|}{ CRC-16 } \\
\cline { 2 - 5 } & Encoder & Decoder & Encoder & Decoder \\
\hline $\begin{array}{c}\text { \# of slice } \\
\text { LUT's }\end{array}$ & 8 & 111 & 16 & 174 \\
\hline \# of slices & 22 & 28 & 32 & 41 \\
\hline $\begin{array}{c}\text { Max Freq. } \\
\text { (MHz) }\end{array}$ & 792.14 & 274.45 & 792.14 & 250.156 \\
\hline
\end{tabular}

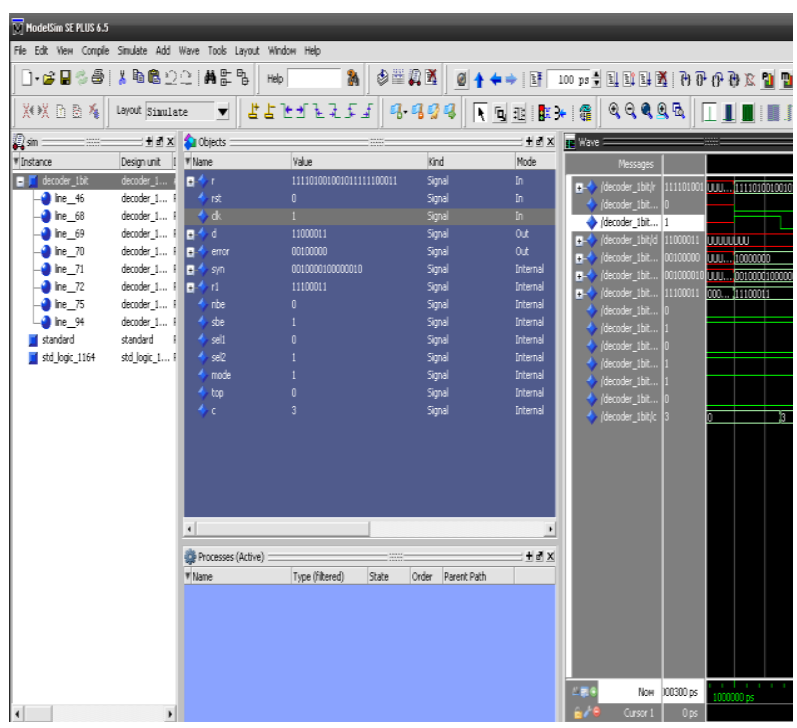

Fig 5: Modelsim Simulation

\section{CONCLUSIONS}

CRC is the method that can detect the error in transferring data between two points. In this paper a new method based on
$\mathrm{CRC}$ has been introduced for single bit error correction. We have implemented on FPGA, for two different standards CRC-8 and CRC-16. We have shown that hardware implementation on FPGA can be effectively used to improve the performance of CRC calculations.

Usually components, like shift registers, XOR and NOR gates are used, so the entire circuit can be easily designed. This approach is efficient both in terms of hardware and speed. The additional hardware required is very simple. This technique works efficiently in case of ASIC design also.

The hardware requirements are tabulated and the algorithm is tested for all possible inputs using a test bench and the algorithm performs as designed.

\section{REFERENCES}

[1] Shukla S, Bergmann N W. "Single bit error correction implementation in CRC-16 on FPGA" In: IEEE International Conference on Field-programmable Technology. Brisbane,Australia, 2004: 319-322.

[2] PAN Yun, GE Ning, DONG Zaiwang. "CRC Look-up Table Optimization for Single-Bit Error Correction". In: TSINGHUA SCIENCE AND TECHNOLOGY ISSN 1007-0214 18/19 pp620-623 Volume 12, Number 5, October 2007.

[3] Johnston C A, Chao H J. "The ATM layer chip: An ASIC for BISDN applications". IEEE Journal on Selected Areas in Communications, 1991, 9(5): 741-750.

[4] Peterson, W. W. and Brown, D. T. "Cyclic Codes for Error Detection "Proceedings of the IRE 49: 228. doi: 10.1109/JRPROC.1961.287814, January 1961.

[5] W.W Peterson, E. J. Weldon. "Cyclic Codes for Error Detection".Second Edition Published by MIT Press, 1972 ISBN 0262160390,9780262160391.

[6] Norman Matloff. "Cyclic Redundancy Checking".

[7] "Double Bits Error Correction Using CRC Method" by Shahram Babaie, Ahmad Khadem Zadeh, Seyed Hasan Es-hagi, Nima Jafari Navimipour.IN: Semantics, Knowledge and Grid, 2009. SKG 2009. Fifth International Conference. Oct. 2009, $254-257,11050651$.

[8] "FPGA implementation of one and two bit error correction using CRC" by Pramod S P, Akshay S kotain, Rajagopal A in International conference on recent trends in computer science and engineering 2012,ISBN 978-819089-807-2.

[9] Digital and analog communication systems, K. Sam Shanmugam, John Wiley, 1996.

[10] Giureppe Campobello, Giuseppe Patane. Marco Russo, "Parallel CRC Realization".

[11] T. V. Ramabadran and S. S. Gaitonde. "A tutorial on CRC computations". IEEE Micro, Vol. 8, No. 4, 1988, pp. 62-75.

[12] T. B. Pei and C. Zukowski "High-speed Parallel CRC Circuits in VLSI" IEEE Tran.on Communications,Vol. 40, April, 1992, pp 635-657 